\title{
Numerical equivalence of advection in flux and advective forms and quadratically conservative high-order advection schemes
}

\author{
Ming Xue ${ }^{1,2}$ and Shian-Jiann Lin $^{3}$
}

\author{
School of Meteorology ${ }^{1}$ \\ Center for Analysis and Prediction of Storms ${ }^{2}$ \\ University of Oklahoma \\ Norman OK 73019 \\ NASA, Goddard Space Flight Center ${ }^{3}$ \\ Greenbelt, MD 20771
}

(Submitted to Mon. Wea. Rev. as a Note)

April 6, 2000

Revised July 20, 2000

Accepted July 28, 2000

Corresponding Author Address:

Dr. Ming Xue, School of Meteorology,

University of Oklahoma, 100 East Boyd, Norman, OK 73019.

E-mail:mxue@ou.edu. 


\begin{abstract}
In finite difference representations of the conservation equations, the flux form of the advection terms is often preferred to the advective form because of the immediate conservation of advected quantity. The scheme can be designed to further conserve higher order moments, e.g., the kinetic energy, which is important to the suppression of nonlinear instability. It is pointed out here that in most cases, an advective form that is numerically equivalent to the flux form can be found, for schemes based on centered difference. This is also true for higher-order schemes and is not restricted to a particular grid type. An advection scheme that is fourth-order accurate in space for uniform advective flows is proposed that conserves both first and second moments of the advected variable in a nonhydrostatic framework. The role of the elastic correction term in addition to the pure flux term in compressible nonhydrostatic models is also discussed.
\end{abstract}




\section{Introduction}

In finite difference representations of the conservation equations (e.g., for momentum, mass, heat and other substances), the flux form of advection terms is more commonly used in primitive equation models because of the immediate conservation of the advected quantities (e.g., momentum, mass and water substances). The scheme can be designed to further conserve higher order moments (here the moments are multiplied by the density in a nonhydrostatic system and by water depth in the case of shallow water equations), e.g., the kinetic energy, which is important to the control of nonlinear instability. Second-order discretization schemes that conserve both the first (e.g., momentum) and the second moment (e.g., kinetic energy) of the advected quantities can be easily formulated in flux form (e.g., Lilly 1964). In general, numerical schemes that conserve more quantities that are conserved by the continuous counterpart perform better, especially in maintaining correct energy cascade in the discrete system. Arakawa (1966) and Arakawa and Lamb (1977) showed for hydrostatic systems that the conservation of both kinetic energy and enstrophy (vorticity squared) by advection schemes prevent systematic and unrealistic energy cascade towards high wave numbers, a cause of nonlinear instability (Phillips 1959). Their pioneering work has since been extended and generalized by many researchers, examples include Sadourny (1972), Arakawa and Lamb, (1981), Mesinger (1981), and Janjic (1984). The schemes are all presented in hydrostatic frameworks in which the flows are quasi-two-dimensional. Numerical schemes that conserve enstrophy are much harder to design in three-dimensional nonhydrostatic systems. Still, the conservation of kinetic energy and the second moments of scalar variables is also highly desirable in nonhydrostatic systems.

For the above reasons, most contemporary nonhydrostatic numerical models use conservative discretization schemes based on the flux-form advection formulation (e.g., Clark 1977; Anthes and Warner 1978; Xue and Thorpe 1991). The advective form is nonetheless also used in some models (e.g., Klemp and Wilhelmson 1978, referred to as KW78 hereafter) partly due to its simplicity when implementing higher-order (e.g., 4th-order) schemes. It should be pointed out that it is certainly possible to design conservative discretization schemes based on the advective form. Early examples can be found in Grammeltvedt (1969), Janjic (1984), Rancic (1988), among others. Most of these schemes were designed for quasi-two-dimensional hydrostatic flows.

Without special care, discretization schemes based on the advective form of advection terms usually do not conserve even the first moment, e.g., the momentum. This is the primary reason why many modelers abandon the use of advective form (e.g., Wilhelmson and Chen 1982, WC82 hereafter; Tripoli and Cotton 1982, TC82 hereafter). Elastic (compressible) meteorological models usually do not predict the full air density using exact mass conservation equation (KW78; TC82). To cast the advection term in an elastic system in flux form, a correction term due to compressibility (to be referred to as the elastic correction term hereafter) is present (or neglected by some modelers) in addition to the pure flux term (e.g., WC82; Dudhia 1993). It is pointed out in this note that there exists, in most cases, a numerically equivalent advective form to a flux form formulation, and neglecting the elastic correction term does not necessarily improve the conservation of the second moment. One of such equivalent advective forms is used by Skamarock and Klemp (1992), but its exact equivalence to a flux form is not well recognized, at least in the nonhydrostatic modeling community. Such equivalence appears to be implicitly recognized by Janjic (1984) and Rancic (1988), for shallow water equations formulated on Arakawa E-grid. 


\section{The numerical equivalence}

Without loss of generality, we consider only 2-D equations for $u$ and $w$ and an equation for the scalar potential temperature $\theta$ in a nonhydrostatic elastic system, and concentrate on the advective part of the equations, which are written as

$$
\begin{aligned}
& u_{t}+u u_{x}+w u_{z}=f_{u}, \\
& w_{t}+u w_{x}+w w_{z}=f_{w}, \\
& \theta_{t}+u \theta_{x}+w \theta_{z}=f_{\theta},
\end{aligned}
$$

where the subscripts $\mathrm{x}$ and $\mathrm{z}$ denote partial differentiations. $f_{u}, f_{w}$ and $f_{\theta}$ represent the right hand side (r.h.s.) of the $u, w$ and $\theta$ equations respectively, and their detailed form varies with applications. The other variables have their usual meanings.

The advection terms in Eqs.(1) are in advective form. In models that do not explicitly predict full air density, the above equations are often multiplied by a base state density $\bar{\rho}(z)$ and recast into a flux form,

$$
\begin{aligned}
& u^{*}+\left[\left(u^{*} u\right)_{x}+\left(w^{*} u\right)_{z}\right]-\left\{u\left(u^{*}{ }_{x}+w^{*}{ }_{z}\right)\right\}=f_{u}, \\
& w^{*}{ }_{t}+\left[\left(u^{*} w\right)_{x}+\left(w^{*} w\right)_{z}\right]-\left\{w\left(u^{*}{ }_{x}+w^{*}{ }_{z}\right)\right\}=f_{w}, \\
& (\bar{\rho} \theta)^{*}{ }_{t}+\left[\left(u^{*} \theta\right)_{x}+\left(w^{*} \theta\right)_{z}\right]-\left\{\theta\left(u^{*}{ }_{x}+w^{*}{ }_{z}\right)\right\}=f_{\theta},
\end{aligned}
$$

where $u^{*} \equiv \bar{\rho} u$ and $w^{*} \equiv \bar{\rho} w$. Systems similar to Eqs.(2) are widely used in elastic cloud models (e.g., TC82). Note that in an anelastic system, the continuity equation is

$$
u^{*}{ }^{*}+w^{*}{ }_{z}=0,
$$

the terms in curly brackets in Eqs.(2) the vanish. In the rest of this paper, we should refer to these terms as the elastic correction terms.

Adopting the following notations:

$$
\begin{aligned}
& \Phi^{n x}=\left(\phi_{i+n / 2}+\phi_{i-n / 2}\right) / 2 \\
& \delta_{n x} \phi=\left(\phi_{i+n / 2}-\phi_{i-n / 2}\right) /(n \Delta x)
\end{aligned}
$$

a second-order centered difference form of Eqs.(2) on the Arakawa C-grid can be written:

$$
\begin{aligned}
& u^{*}{ }_{t}+\left[\delta_{x}\left(\overline{u^{*}} \bar{u}^{x}\right)+\delta_{z}\left(\overline{w^{*}} x \bar{u}^{z}\right)\right]-\left\{u\left(\delta_{x} \overline{u^{*}} x+\delta_{z} \overline{w^{*}} x\right)\right\}=f_{u}, \\
& w^{*}{ }_{t}+\left[\delta_{x}\left(\overline{u^{*}} \bar{w}^{x}\right)+\delta_{z}\left(\overline{w^{*}} z \bar{w}^{z}\right)\right]-\left\{w\left(\delta_{x} \overline{u^{*}} z+\delta_{z} \overline{w^{*}} z\right)\right\}=f_{w},
\end{aligned}
$$




$$
(\bar{\rho} \theta)^{*}+\left[\delta_{x}\left(u^{*} \bar{\theta}^{x}\right)+\delta_{z}\left(w^{*} \bar{\theta}^{z}\right)\right]-\left\{\theta\left(\delta_{x} u^{*}+\delta_{z} w^{*}\right)\right\}=f_{\theta}
$$

It is well known that for an anelastic flow the advective process in the above system conserves momentum $\bar{\rho} u, \bar{\rho} w$, the kinetic energy $\bar{\rho}\left(u^{2}+w^{2}\right) / 2$, and $\bar{\rho} \theta$ as well as $\bar{\rho} \theta^{2}$ (Lilly, 1964). In a compressible system, the density $(\bar{\rho})$ weighted divergence $\operatorname{Div} 2 \equiv\left(u^{*}{ }_{x}+w^{*}{ }_{z}\right)$ is, though small in general, non-zero, exact conservations on longer exist. WC82 and Ikawa (1988) choose to neglect the elastic correction terms in an attempt to preserve conservations. Doing so does make the first moment conservative. However, without Eq.(3) being satisfied, the second moment is not conservative either (see Appendix). In fact, neglecting them makes the model integration less stable in some cases (Ikawa 1988; Durran and Klemp 1983). Sound wave damping of certain type alleviates its impact (Ikawa 1988; Skamarock and Klemp 1992). In a recent effort to compare the results of the ARPS model (Xue et al. 1995) against that of KW78 model with WC82 formulation (which uses flux-form advection but neglects the elastic correction terms), Richardson (1999) found that a good agreement in the time evolution of a multi-cell convective storm simulation could not be obtained until the neglected elastic correction term is added back to the equation of water vapor mixing ratio in KW78 model. For the comparison purpose, the ARPS code was modified to the maximum extent possible to match the WC92 version of KW78 model, except for the treatment of advection. Certain simple bubble tests also show that the solutions including the elastic correction term are better behaved, in terms of producing more coherent structure of the rising bubble. Ikawa (1988) found that his mountain flow solution becomes unstable when the horizontally explicit and vertically implicit mode-split time integration scheme (as used in ARPS and WC82) is used together with the flux-form advection without elastic correction, unless extra effort is made to damp deep horizontal acoustic modes. These results suggest strongly that the elastic correction terms should not be neglected. follows

Finite differencing can be based on the advective form of Eqs.(2), which are then discretized as

$$
\begin{aligned}
& u^{*}{ }_{t}+{\overline{\left({\overline{u^{*}}}^{x} \delta_{x} u\right)}}^{x}+{\overline{\left.\overline{w^{*} x} \delta_{z} u\right)}}^{z}=f_{u}, \\
& w^{*}{ }_{t}+{\overline{\left(u^{* z} \delta_{x} w\right)}}^{x}+{\overline{\left.\overline{w^{*}} \delta_{z} w\right)}}^{z}=f_{w}, \\
& (\bar{\rho} \theta)^{*}+{\overline{\left(u^{*} \delta_{x} \theta\right.}}^{x}+{\overline{\left(w^{*} \delta_{z} \theta\right.}}^{z}=f_{\theta} \text {. }
\end{aligned}
$$

Similar finite differencing is done in Skamarock and Klemp (1992). However, it is not generally recognized that Eqs. (6) are exactly equivalent to Eqs. (5). It takes only some algebraic manipulations to prove for the $u$ equation, for instance, that

$$
\begin{aligned}
& \left.{\left.\overline{\left(\bar{u}^{*} x\right.} \delta_{x} u\right)}^{x} \equiv \delta_{x}{\overline{u^{*}}}^{x} \bar{u}^{x}\right)-u \delta_{x}{\overline{u^{*}}}^{x}, \\
& {\left.\overline{\left(w^{* x}\right.} \delta_{z} u\right)}^{z} \equiv \delta_{z}\left({\overline{w^{*}}}^{x} \bar{u}^{z}\right)-u \delta_{z}{\overline{w^{*}}}^{x} .
\end{aligned}
$$


Eq. (5a) and Eq. (6a) are therefore exactly the same. Hence, there is no need to split the advection term into two parts to take advantage of the known conservation properties of flux form. From a practical point of view, formulating the term in advective form cuts the computations by half, and in addition, round-off errors are also reduced simply because there are less floating-point operations. In an anelastic system where Eq. (3) is exactly satisfied, the equivalence between the two systems also holds. The same is true for other systems whose flux formulation of advection terms does not involve a correction term.

Numerical experiments were performed to verify the above observations. We have formulated a 3-D compressible model using the split-explicit time integration scheme of KW78. Two runs with formulations (5) and (6) are found, without any explicit diffusion, to be stable for long time integrations, and the results are essentially identical (with only difference being caused by machine round-off errors). In contrast, when the advection terms were formulated, e.g. for the u equation, in a straightforward fashion on a C-grid as

$$
u^{*} \delta_{x} \bar{u}+\overline{w^{*}} x z \delta_{z} \bar{u}
$$

explicit numerical diffusion must be added to control nonlinear instability. Without diffusion, the model run blows up after certain time.

\section{Extension to higher-order and other grid structures}

The equivalent advective form can also be found for fourth-order differencing schemes as well as for schemes based on other types of grid structure. For example, for the u equation, a fourth-order accurate difference scheme on the Arakawa C-grid can be formulated by a straightforward extension of the conservative second-order scheme:

$$
\begin{aligned}
& \left\{\frac{4}{3}\left[\delta_{x}\left({\overline{u^{*}}}^{x} \bar{u}^{x}\right)+\delta_{z}\left({\overline{w^{*}}}^{x} \bar{u}^{z}\right)\right]-\frac{1}{3}\left[\delta_{2 x}\left({\overline{u^{*}}}^{2 x} \bar{u}^{2 x}\right)+\delta_{2 z}\left(\bar{w}^{* x z} \bar{u}^{2 z}\right)\right]\right\}-u \overline{\operatorname{Div} 4}^{x} \\
& \equiv \frac{4}{3}\left[{\overline{\left({\overline{u^{*}}}^{x} \delta_{x} u\right)}}^{x}+{\left.\overline{\left(\bar{w}^{*}\right.} \delta_{z} u\right)}^{z}\right]-\frac{1}{3}\left[{\overline{\left(\bar{u}^{* 2 x} \delta_{2 x} u\right)}}^{2 x}+{\overline{\left(\bar{w}^{* x z} \delta_{2 z} u\right)}}^{2 z}\right],
\end{aligned}
$$

and for scalar $\theta$,

$$
\begin{aligned}
& \left\{\frac{4}{3}\left[\delta_{x}\left(u^{*} \bar{\theta}^{x}\right)+\delta_{z}\left(w^{*} \bar{\theta}^{z}\right)\right]-\frac{1}{3}\left[\delta_{2 x}\left(\bar{u}^{*} x \bar{\theta}^{2 x}\right)+\delta_{2 z}\left(\bar{w}^{* z} \bar{\theta}^{2 z}\right)\right]\right\}-\theta \operatorname{Div} 4 \\
& \equiv \frac{4}{3}\left[{\overline{\left(u^{*} \delta_{x} \theta\right.}}^{x}+{\overline{\left(w^{*} \delta_{z} \theta\right.}}^{z}\right]-\frac{1}{3}\left[{\overline{\left(\bar{u}^{* x} \delta_{2 x} \theta\right)}}^{2 x}+{\overline{\left(\bar{w}^{* z} \delta_{2 z} \theta\right.}}^{2 z}\right],
\end{aligned}
$$

where the fourth-order (base state) density weighted divergence Div4 is defined at the scalar point as

$$
\operatorname{Div} 4 \equiv \frac{4}{3}\left[\left(\delta_{x} u^{*}+\delta_{z} w^{*}\right)\right]-\frac{1}{3}\left[u\left(\delta_{2 x} \overline{u^{*}}+\delta_{2 z} \overline{w^{*}}\right)\right]
$$


Terms on the left hand side of Eqs.(9) and (10) are in flux form whereas those on the r.h.s. are in the equivalent advective form. In an anelastic system where Div4 in (11) is identically zero, the fourth-order advections in both forms conserve first and second moments (see appendix for proof) of the advected quantities. Again neglecting the Div4 term on the left hand side to yield a pure flux term, as is done in WC82, does not guarantee the conservation of second moments in a compressible model.

It should also be noted that the formulations in Eqs. (9) and (10) are only quasi-fourth-order accurate, mainly because the linear spatial average is introducing second-order truncation errors that do not cancel each other. High-order spatial averaging must be used to construct a truly fourth-order scheme, and more grid points will be involved in such a scheme. The fourth-order scheme used by WC82 is not truly fourth-order for non-constant advective flow either. Both schemes reduce to the same truly fourth-order scheme when the flow is uniform in space. The scheme in flux form as given in Eqs. (9) and (10) has been implemented in the model of Xue and Thorpe (1991), and the Advanced Regional Prediction System (Xue et al. 1995), and the experiments with a cold downburst/density current flow showed superior solutions to those obtained using the second-order counterparts as given in Eqs. (5) and (6).

Equivalent advection and flux forms based on centered difference can also be found for other types of grid structure, such as the Arakawa B-grid used in the nonhydrostatic version of the Penn. State/NCAR mesoscale model (Dudhia 1993). The key step is to perform proper spatial averages on the advection terms as exemplified in Eqs. (6), (9) and (10), instead of directly evaluating them at the point of the predicted variable [as in Eq. (8)]. Such spatial averaging has the desired de-aliasing effect through the conservation of first and second moments. Since it is not the purpose of this note to exhaust all possibilities, we will not go into more details here.

\section{Summary}

In this note, we have pointed out that the flux form of the advection terms in conservation equations can, for most schemes based on centered finite-difference, have a numerically equivalent formulation in advective form. This equivalent advective form has the same conservation properties as the corresponding flux form. Such equivalence can also be found for higher-order (=4) schemes and on grid structures other than the Arakawa $\mathrm{C}$ type. These schemes are (nearly) conservative up to the second moments in the absence of (with weak) compressibility. The advective form is generally simpler than the flux form counterpart. In certain compressible atmospheric models, in which an elastic correction term is needed in addition to the pure flux term that includes a base-state density, using the equivalent advective form can save up to half of the computational time. It is also pointed out that neglecting the elastic correction term for the sake of momentum conservation, as is done in at least one nonhydrostatic atmospheric model, is undesirable.

Acknowledgments. This work was supported by NSF through ATM-8809862 to the Center for Analysis and Prediction of Storms (CAPS), University of Oklahoma, and by NSF ATM-9909007 to the first author. The authors wish to thank Dr. A. Shapiro for reading the original manuscript. Most of the work described in this paper was conducted and a draft manuscript completed in the early 1990's when both authors were working at CAPS. Two anonymous reviewers are thanked for their constructive comments. 


\section{APPENDIX}

\section{Conservation properties of the terms in Eqs.(9) and (10)} becomes

Formulating the advection terms in the fourth-order flux form as given in Eq.(9), the urequation

$$
\begin{aligned}
\frac{? u^{*}}{? t}= & -\left\{\frac{4}{3}\left[\delta_{x}\left(\bar{u}^{*} \bar{u}^{x}\right)+\delta_{z}\left(\bar{w}^{*} x \bar{u}^{z}\right)\right]\right. \\
& \left.\left.-\frac{1}{3}\left[\delta_{2 x}\left(\bar{u}^{2 x} \bar{u}^{2 x}\right)+\delta_{2 z}{\overline{w^{*}}}^{x z} \bar{u}^{2 z}\right)\right]\right\}+u \overline{\text { Div4 }}{ }^{x}+\text { other terms. }
\end{aligned}
$$

Multiplying (A1) by u, rearranging the terms and summing the equation over the entire model domain yields

where

$$
\begin{aligned}
& \frac{?}{? t}\left[\sum_{i j}\left(\bar{\rho}_{i, j} u_{i, j}^{2} / 2\right)\right]= \\
& -\sum_{i j}\left\{\frac { 4 } { 3 } \left[\left(\tilde{u}_{i+1 / 2, j}^{*} u_{i+1, j} u_{i, j}-\tilde{u}^{*}{ }_{i-1 / 2, j} u_{i, j} u_{i-1, j}\right) /(2 \Delta x)+\right.\right. \\
& \left.\left(\tilde{w}^{*}{ }_{i, j+1 / 2} u_{i, j+1} u_{i, j}-\tilde{w}^{*}{ }_{i, j-1 / 2} u_{i, j} u_{i, j-1}\right) /(2 \Delta z)\right] \\
& -\frac{1}{3}\left[\left(\hat{u}^{*}{ }_{i+1, j} u_{i+2, j} u_{i, j}-\hat{u}^{*}{ }_{i-1, j} u_{i, j} u_{i-2, j}\right) /(4 \Delta x)+\right. \\
& \left.\left.\left(\hat{w}^{*}{ }_{i, j+1} u_{i, j+2} u_{i, j}-\hat{w}^{*}{ }_{i, j-1} u_{i, j} u_{i, j-2}\right) /(4 \Delta z)\right]\right\} \\
& -\frac{1}{2} \sum_{i j}\left(u_{i, j}^{2} \overline{\operatorname{Div} 4}^{x}\right)+\sum_{i j}\left(u_{i, j}^{2} \overline{D i v 4}^{x}\right)+\text { other terms }
\end{aligned}
$$

$$
\begin{array}{ll}
\tilde{u}^{*}{ }_{i+1 / 2, j} \equiv\left(u_{i+1, j}^{*}+u^{*}{ }_{i, j}\right) / 2, & \tilde{w}^{*}{ }_{i, j+1 / 2} \equiv\left(w_{i, j+1}^{*}+w_{i, j}^{*}\right) / 2, \\
\hat{u}^{*}{ }_{i+1, j} \equiv\left(u^{*}{ }_{i+2, j}+u^{*}{ }_{i, j}\right) / 2, & \hat{w}^{*}{ }_{i, j+1} \equiv\left(w_{i, j+2}^{*}+w_{i, j}^{*}\right) / 2 .
\end{array}
$$

In (A2), The terms inside the first summation on the r.h.s. of Eq.(A2) are of the form $\left(A_{i+1 / 2}-A_{i-1 / 2}\right)$ or $\left(A_{i+1}-A_{i-1}\right)$, thus all terms except those on the boundary cancel, generating no net energy in the interior domain. The second summation term on the r.h.s. originates from the pure flux term in Eq.(9) and the third summation term comes from the divergence term in the same equation. Both terms represent energy sources when Div4 is not zero. As pointed out previously in the text, even if the Div4 term in Eqs.(9) or (10) [hence the third summation on the r.h.s. of Eq.(A2)] is neglected, the conservation of second moments is not guaranteed. On the other hand when Div4 is zero (or very small), the above formulation possesses an exact (or a good) conservation (of the second moment). Finally, the conservation properties of $w$ and scalar equations can be considered in a similar fashion. When the second moments of all three velocity components are conserved, so is the kinetic energy. The conservation of the first moment by Eq.(A1) is evident when Div4=0. 


\section{REFERENCES}

Anthes, R. A. and T. T. Warner, 1978: Development of hydrodynamic models suitable for air pollution and other mesometeorological studies. Mon. Wea. Rev., 106, 1045-1078.

Arakawa, A., 1966: Computational design for long-term numerical integrations of the equations of atmospheric motion. Part I: Two dimensional incompressible flow. J. Comp. Phy., 1, 119-143.

Arakawa, A., and V. R. Lamb, 1977: Computational design of the basic dynamical processes of the UCLA general circulation model. In: J. Chang (Editor), Methods in Computational Physics. Academic Press, 174-264.

Arakawa, A., and V. R. Lamb, 1981: A potential enstrophy and energy conserving scheme for the shallow water equations. Mon. Wea. Rev., 109, 18-36.

Clark, T. L., 1976: A small scale dynamic model using a terrain-following coordinate transformation. $J$. Comput. Phys., 24, 186-215.

Dudhia, J., 1993: A nonhydrostatic version of the Penn State-NCAR mesoscale model: Validation tests and simulation of an Atlantic cyclone and cold front. Mon. Wea. Rev., 121, 1493-1513.

Durran, D.R., and J.B. Klemp, 1983: A compressible model for the simulation of moist mountain waves. Mon. Wea. Rev., 111, 2341-2361.

Grammeltvedt, A., 1969: A survey of finite-difference schemes for the primitive equation for a barotropic fluid. Mon. Wea. Rev., 97, 384-404.

Ikawa, M., 1988: Comparisons of some schemes for nonhydrostatic models with orography. J. Met. Soc. Japan, 66, 753-776.

Janjic, Z. I., 1984: Nonlinear advection schemes and energy cascade on semi-staggered grids. Mon. Wea. Rev., 112, 1234-1245.

Klemp, J. B. and R. B. Wilhelmson, 1978 (KW78): The simulation of three-dimensional convective storm dynamics. J. Atmos. Sci., 35, 1070-1096.

Lilly, D. K., 1964: Numerical solution for the shape-preserving two-dimensional thermal convection element. J. Atmos. Sci., 21, 83-98.

Mesinger, F., 1981: Horizontal advection schemes of a staggered grid - an enstrophy and energyconserving model. Mon. Wea. Rev., 109, 467-478.

Phillips, N. A., 1959: An example of nonlinear computational instability. In: B. Bolin (Editor), The Atmosphere and the Sea in Motion. Rockefeller Inst. Press in association with Oxford Univ. Press, New York, 501-504.

Rancic, M., 1988: Forth-order horizontal advection schemes on the semi-staggered grid. Mon. Wea. Rev., 116, 1274-1288.

Richardson, Y., 1999: The Influence of Horizontal Variations in Vertical Shear and Low-Level Moisture on Numerically Simulated Convective Storms. Ph.D. Dissertation. University of Oklahoma [Available from School of Meteorology, University of Oklahoma, 100 E. Boyd, Norman OK 73019].

Sadourny, R., 1972: Conservative finite-difference approximations of the primitive equations on quasiuniform spherical grids. Mon. Wea. Rev., 100, 136-144.

Skamarock, W. C., and J. B. Klemp, 1993: Adaptive grid refinement for two-dimensional and threedimensional nonhydrostatic atmospheric flow. Mon. Wea. Rev., 121, 788-804. 
Tripoli, G.J. and W.R. Cotton, 1982 (TC82): The Colorado State University three-dimensional cloud/masoscale model - 1982. Part I: General theoretical framework and sensitivity experiments. J. Rech. Atmos., 16, 185-220.

Wilhelmson, R.B. and C.-S. Chen, 1982 (WC82): A simulation of the development of successive cells along a cold outflow boundary. J. Atmos. Sci., 39, 1466-1483.

Xue, M. and A. J. Thorpe, 1991: A mesoscale numerical model using the nonhydrostatic sigmacoordinate equations: Model experiments with dry mountain flows, Mon. Wea. Rev., 119, $1168-1185$.

Xue, M., K. K. Droegemeier, V. Wong, A. Shapiro, and K. Brewster, 1995: ARPS Version 4.0 User's Guide. 380 pp. [Available from CAPS, University of Oklahoma, 100 E. Boyd St., Norman OK 73019. Also at http://www.caps.ou.edu/ARPS.] 
Office of Chief Editors, Monthly Weather Review

Attn: Editorial Assistant

FL3-Rm 3075

3450 Mitchell Lane, Boulder, CO 80301

Boulder CO 80307-3000

Tel: 303-497-8936

\section{Dear Editor,}

Enclosed are fives copies of manuscript entitled "Numerical equivalence of advection in flux and advective forms and quadratically conservative high-order advection schemes" by myself and Shian-Jiann Lin. It is intended for the Notes and Correspondence Section. The material has not been published or submitted for publication elsewhere. The copyright transfer form will be submitted later. I appreciate your considering the paper for publication in the Monthly Weather Review.

Sincerely,

Ming Xue 
Center for Analysis and Prediction of Storms

University of Oklahoma

100 E. Boyd

Norman OK 73019

Office of Chief Editors, Monthly Weather Review

Attn: Editorial Assistant

FL3-Rm 3075

3450 Mitchell Lane, Boulder, CO 80301 
Dr. William Skamarock

Editor, Monthly Weather Review

NCAR, P. O. Box 3000

Boulder CO 80307-3000

Tel: 303-497-8893

Dear Editor,

Enclosed are four copies of revised manuscript entitled "Numerical equivalence of advection in flux and advective forms and quadratically conservative high-order advection schemes" by myself and Shian-Jiann Lin, and a copy of our responses to the reviewers.

The copyright transfer form is also enclosed. I appreciate your consideration for the paper to be published in the Monthly Weather Review.

Sincerely,

Ming Xue

School of Meteorology

University of Oklahoma 
School of Meteorology

University of Oklahoma

SEC 1310, 100 E. Boyd

Norman OK 73019

Dr. William Skamarock

Editor, Monthly Weather Review

NCAR, P. O. Box 3000

Boulder CO 80307-3000

Tel: 303-497-8893 
Dr. Ming Xue

School of Meteorology

University of Oklahoma

100 East Boyd

Norman, OK 73019

Dear Dr. Xue:

I have received two reviews for your manuscript entitled:

Title: Numerical equivalence of advection. in flux and advective forms and quadratically conservative high-order advection schemes

Author: Ming Xue and Shian-Jiann Lin

Manuscript: $689 \mathrm{KS} 20$

which you have submitted to MWR. I find that this paper may be suitable for publication in Monthly Weather Review, on the condition that revisions are performed. The suggested revisions would improve the manuscript to an acceptable form. If adequate revision is not performed, then this paper may be found unacceptable for publication and rejected.

Please pay close attention to the comments of Reviewer B, particularly those pertaining to conservations properties of the discreet versus the continuous equations; the reviewer correctly points out that the continuous equations conserve mass, momentum, and energy regardless of their form (flux or advective), while the same cannot necessarily be said of the discreet forms. Reviewer B also notes that the advective form discretizations can be made mass and momentum conserving (along with the enstrophy and energy conservation often built in to the hydrostatic advective-form models. Also, a more evenhanded treatment of the results coming from the hydrostatic modeling community would be needed in the revised manuscript.

Please examine each reviewer's comments carefully and provide a point-by-point response to each stating what was done or why the comment was not addressed.

When submitting your revised manuscript, please return four (4) copies of the revised manuscript and a point-by-point response to the reviewer's comments. You will also need to address those items (if any) indicated on the Manuscript Evaluation form before final acceptance. Please note that the AMS requires a revised manuscript to be submitted within four (4) months.

Thank you for submitting your manuscript to the Monthly Weather Review. I look forward to receiving your revised manuscript and response to the reviewers' comments.

Sincerely,

William C. Skamarock

Editor

Enclosures

Monthly Weather Review 


\section{Response to Review A (responses are in italic):}

Review of "Numerical equivalence of advection in flux and advective forms and quadratically conservative high-order advection schemes"

by M. Xue and S.-J. Lin

Recommendation: Accept with minor revision

Despite accepting this paper with minor revisions because its content is clear, and fairly accurate, with only little editing required for English, I have reservations as to the originality of these ideas. The authors suggest that it is not "generally recognized" that certain advective forms with divergence terms and flux terms have an exact equivalence to other advective forms in the finite difference equations. It depends on the meaning of 'generally recognized", and I suspect it -is - at least widely recognized. If that is the case, the paper presents I little that is new to readers except for the extension to 4th order that may be less well known. For me, it is only this extension that makes the Note acceptable, but only barely because it is a very minor contribution to the knowledge in the field.

It does appear that the equivalence of the flux and advective formulations is recognized by some modelers. It is probably also correct to say that the equivalence has not been stated explicitly in the literature. We do view the fourth-order extension of the quadratically conservative formations in either flux or advection form as an important element in this paper, as is suggested by this reviewer. While forth-order advection formations that conserve kinetic energy as well as enstrophy have been proposed in several past papers, we are not aware of the current forthorder formulation being used elsewhere. Most of the early schemes based on stream-and potential functions may not be directly applicable to nonhydrostatic flows that tend to be fully three dimensional.

Furthermore no attempt is made to clarify exactly why using the flux form without a divergence term leads to noisier results in compressible models. It is just stated as a fact. Presumably sound wave aliasing is playing a role here. Since Ikawa (1988) has previously stated these points about noisiness this part is not new, and some further insight would add value to this Note.

It appears that this reviewer is referring to the discussion on the results of bubble experiments in the paragraph proceeding Eq. (6). We did say that the solutions including the elastic correction term are better behaved, here we actually refer to the structure of the simulated bubble (we have modified the text to reflect this). We added in this paragraph reference to the instability found by Ikawa with flux-form advection without elastic correction, which lends extra support for retaining the correction term or using the advective formulation.

Specific Points

1. p4. Top line. "however, ..." should probably start a new sentence.

Done.

2. p4. WC92. References to WC92 should be WC82. 


\section{Corrected.}

3. p6, eq (11). Extraneous theta and $u$ in these terms.

\section{Corrected.}

4. p9-10. References to eqs (8) and (9) should be to (9) and (10), including in section title.

\section{Corrected.}

5. p10. Minor note: I think it would have been clearer to first state that the second summation term comes from the flux term, and the third summation term comes from the Div4 term in eqs (9) and (10), then go on to make points about the energy sources.

Sentences modified as suggested. Thanks. 


\section{Response to Review B (responses are in italic):}

Review of the manuscript

Numerical equivalence of advection in flux and advective forms and quadratically conservative high-order advection schemes

by Ming Xue and Shian-Jiann Lin

submitted to the Monthly Weather Review

Recommendation: Revision. Possibly the authors want to reconsider submitting the manuscript in view of the variety of problems with the points they are making?

Significant revisions have been done. See details below.

The problem with this note is that the authors are obviously not aware that points they are making tend to have been made already albeit perhaps implicitly and in the context of hydrostatic models; or are not valid beyond the community the authors are familiar with. Reading the abstract and introduction I could not avoid the feeling that we - the weather prediction modeling community -- consist of two somewhat distinct groups of people: those who came to the field through the early general circulation and synoptic-scale weather prediction efforts (including the "Arakawa" subgroup: Arakawa, Sadourny, Mesinger, Janjic, Rancic, ...) and those who have come to the field somewhat later from the small-scale cloud modeling/mountain waves work. People from the latter group. I suppose felt little need to have a look into what the former group -- the mentioned subgroup in particular.

We agree that the original manuscript gave too little recognition of many significant work done by the 'Aarakawa" subgroup. We would not say, however, that the authors felt little need to study work performed by this group. Without doubt, this work sits on the foundation of these important early work and the authors hold great respect for these pioneers. In the revised version, many of the papers are duly recognized and their relevance to the current paper discussed where appropriate.

To be specific, let me start with the first sentence of the abstract: "In finite difference representation of the conservation equations the flux form of the advection terms is generally preferred to the advective form because of its conservation properties". I have two problems with this sentence.

The first: I disagree that the flux form indeed "is generally preferred". I see little room for doubt that within the Arakawa-type "conservation" community, it is the advective form which was and is generally preferred, In Grammeltvedt (MWR 1969) survey of ten shallow water schemes five of them are both. conservative schemes of various 
kinds and are in advective form. Sadourny (JAS, April 1975) is using advective forms (the scheme used in the ECMWF's grid-point model, later in HIRLAM), as well as Janjic (Contr. Atmos. Phys., 1977), Mesinger (MWR, March 1981), Janjic (MWR, June 1984) and Rancic (MWR, June 1988).

We agree that the statement in the original manscript may be too strong. It appears that certain authors do prefer the advection form of equations (e.g., Janjic 1984; Rancic 1988). However, flux-form finite difference formulations are preferred in the nonhydrostatic modeling community because they immediately conserve the first moment. Conservation of first moments is even more important in climate simulations. We believe it is for this reason, most grid-point global hydrostatic models currently in use (e.g., UCLA, NASA Goddard, GFDL, and CSU) use flux-form (not advective form) for transport of scalars (e.g, water substances, potential temperature, and surface pressure). To conserve higher order moments and/or quantities like enstrophy, careful design is needed for either advective or flux formlation.

Both the abstract and the text (mostly in the Introduction) have been revised to communicate this more accurately. References to papers that use advective formulations are added.

Fairly straightforward tricks were used for decades to come up with conservation schemes while not abandoning the simplicity of the advective form. Rancic (1988, p. 1278, Eq. 23-24) can be used for illustration: definitions of $\mathrm{h}$ sub $\mathrm{u}$ and $\mathrm{h}$ sub $\mathrm{v}$ are worked out to achieve the desired end. I do not recall if someone had pointed out as a major discovery the 'equivalence of advection in flux and advective forms', but I'd say this equivalence was obvious to those of us who have gone through various exercises published in the cited papers, since one must build a flux-form-like time difference equation (time change of the product $h$ times the quantity to be conserved) in order to demonstrate conservation. In other words, what we would use in advective form was clearly equivalent to the flux form to which we had to go back to to demonstrate conservation.

It does appear that the equivalence of the flux and advective formulations is recognized by some modelers, including this reviewer. It is probably also correct to say that such recognition has not been stated explicitly in the literature. We view our main contribution here to be in the context of nonhydrostatic models. While forth-order advection formations that conserve kinetic energy as well as enstrophy have been proposed in the cited papers, we are not aware of the current forth-order formulation being used elsewhere.

My second problem with the first sentence is with: 'flux form ...

preferred ... because of its conservation properties*. Flux forms of the equations are obtained from their advective form and are therefore equivalent to them; the two have the same conservation properties. Thus, what special conservation properties does one have in mind here? There are none. 
Perhaps what the authors want to say is that in the community such and such people have preferred the flux form because of the straightforwardness of arriving at conservation schemes when the flux form is used. Or, because of being aware that the advective form is just about as good to design conservation schemes, with an extra benefit of the simplicity of use. Or still, *... because of its perceived conservation properties."

We should have said the immediate conservation of the first moment, by flux form finite difference schemes. The text has been changed. See also earlier comment.

The third and the fourth sentence of the abstract: note that in both Mesinger's and Rancic's cited papers fourth-order schemes are designed conserving various quantities. The difference is, it would not be "the first and the second' moment of the advected quantities, but it would be kinetic energy - the second moment - and enstrophy. The first moment, momentum, is conserved in the schemes of Janjic (1984) and Rancic 1988 fourth-order version of the Janjic 84 scheme). Note that it is not "both the first and the second moment of the advected quantities' that "play a key role in controlling nonlinear instabilities caused by aliasing errors' as discussed by Arakawa and Lamb (the first paragraph of introduction), but that these quantities are kinetic energy and enstrophy. In other words, not the first moment.

The reviewer is correct in pointing out that the conservation of the first moment does not contribute the prevention of nonlinear aliasing, although it is definitely desirable. The conservation of kinetic energy certainly does. It would be nice that enstrophy (in 3D formulation perhaps?) is also conserved in a nonhydrostatic framework but such schemes appear to have yet to be developed. The impact on energy cascade in three dimensional flows does not appear to be well understood, as we do for two-dimensional flows. The text has been revised accordingly.

There are more examples of text that requires attention, The 1st sentence of the 2nd paragraph: "The consensus is that the advective form conserves neither the first nor the second moment.* It is a finite difference scheme that can either conserve something or not conserve- not the advective or the flux form of the continuous equations. Once again, the advective and the flux form of the equations are equivalent and have the same conservation properties.

Again, the original text is misleading. It should have referred to the discrete formulations based on the flux or advection form of conservation equations, not the equations themselves. Revision has been performed. 
Being obviously of the religion the authors advocate I appreciate the work done but for the note to be accepted for publication the shortcomings pointed out need to be addressed.

We appreciate the expert comments by this reviewer. 